\title{
Calibrating Einstein field equations using rippling 3-Riemannian structure for gravity with dark matter effects
}

\author{
Shivam S. Naarayan \\ University of Delhi, New Delhi-11000\%, India
}

(Dated: October 2, 2021)

\begin{abstract}
The paper presents modifications to Einstein field equations (EFEs) based on the model proposed in the working paper, Rippling 3-Riemannian structure describing gravity with dark matter effects. The model proposes matter and energy are separate entities and energy is a property of threedimensional probabilistic structure spanning space. Mass interacts by binding energy density causing variations in length and time scales, mathematically equivalent to spacetime curvature in general relativity. Gravity is thus described as flow and distribution of energy density. Bounded energy density is the additional source of gravity leading to dark matter observations. The results of the model proposes two EFEs for large and largest scales and further predicts dependence of cosmological constant on space and time coordinates.
\end{abstract}

\section{INTRODUCTION}

The paper explores the missing mass problem (dark matter) and proposes modifications to Einstein field equations (EFEs) based on alternate model of gravity presented in the preceding working paper [1]. The proposed model unifies gravity, dark matter and dark energy.

\section{A. Model summary from working paper}

In this paper I employ the novel theoretical framework presented in the working paper [1]. The framework considers matter and energy as separate entities. It assigns energy to a three-dimensional probabilistic structure, Kaal ("dark"), spanning space i.e. space is not featureless. Each point of the structure is defined by (a) Kaal energy density (KED, $\kappa$ ) (b) Time density (c) Metric of Riemannian 3-Manifold $\left(g_{m n}\right)$. Such description leads to emergence of local length and time scales in correspondence with results of general relativity and further predicts common origin for the observed phenomenon attributed to dark energy and dark matter. In case of rotating source mass, additional gravitational effects are predicted to be caused by energy bounded by the source mass (bounded Kaal energy (BKE)) and the flow of energy from equatorial planes to the poles along axis of rotation. The model bears similarity with SFDM model [2, 3] in principle.

The central idea of the model 1 is that variations in local length and time scales (not arbitrary measurements) are symmetric and orthogonal due to energy-momentum relation in special relativity and these variations emerge from flow and distribution of energy density in a local patch of Kaal structure. This is immediate on employing light frequency and wavelength for time and length measurements respectively as speed of light is universally constant. The working paper shows that variation in time scales can be determined by evaluating the variation in length scales. The most used Schwarzchild and Kerr metric comply and any metric solution 23 to EFEs

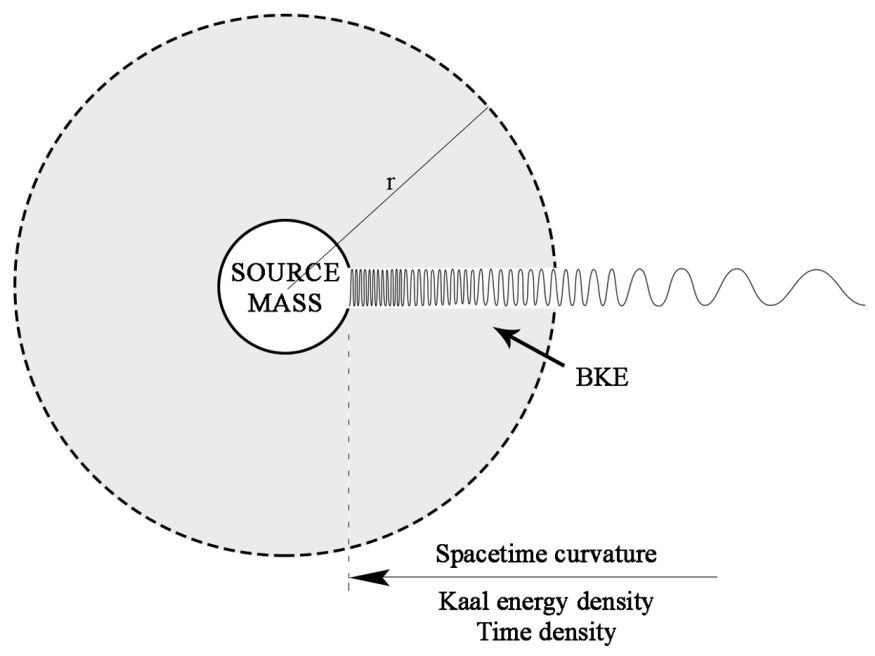

must correspond to the coequality of variation in length and time scales since it emerges from energy-momentum relation in special relativity. The part of spacetime metric in general relativity that reflects non-orthogonality of space and time coordinates i.e. motion of source mass is captured by dragging of Kaal Fluid of energy in the proposed framework.

The model [1] relies on the distinction between scales and measurements; while locally scales are constant, measurements can be arbitrary. The model is about emergence of local scales that enable measurements by an observer. Thus, the present framework complements general relativity and is used to modify the EFEs for large and largest scales to completely describe the physical phenomenon.

\section{B. Conclusions from the model}

\section{Gravity}

In the working paper[1], gravity is proposed to be a consequence of flow and distribution of Kaal ('dark') en- 
ergy density $(\mathrm{KED}, \kappa)$ bounded by source mass.

In general relativity [4, spacetime manifold is description of measurements of length and time; while proper length and proper time are indicative of length and time scales relative to flat conditions. Here, the proposed model is based on coequality of variations in length and time scales which is evident from energy-momentum relations in relativity. Thus, the curvature of spacetime is found to be equivalent to variation in Kaal energy density.

\section{Flat Space}

In the working paper 1, it was also proposed that curvature in spacetime is curvature in measurements of length and time not in physical space. This was made immediately evident by employing light rods and light clock for measurements in which case the scales for length and time measurements squeeze or stretch leading to variations in local measurements. This does not alter the mathematics of GR in weak conditions but only the interpretation. This is possible due to introduction of rippling Kaal structure of energy. Thus, while the local measurements may vary but the space is flat in correspondence with Einstein-deSitter model.

\section{Expansion of Universe}

As discussed in working paper [1, in regions free from matter, Kaal structure tends towards minimization of local energy density. This results in changes in local time density and metric for length measurement. On cosmic scales, expansion is thus a result of minimization of local energy density in intergalactic space. The wavelength of Kaal Ripples (ripples of flow of energy) increases causing the local energy density to fall and pushing the galaxies apart (unless they are caught in the flow of bounded energy density of other cosmic structure). Because the energy density is consistently falling, the expansion rate must also be monotonically decreasing.

\section{Literature Review}

The missing mass problem was first noted by Fritz Zwicky way back in 1930s [5] which led to the understanding that the most mass of galaxy or galaxy clusters is non-luminous eventually leading to the term 'dark matter'. There is undeniable evidence for effects observed under the head 'dark matter' especially on largest scales. There have been two approaches to solve this problem, non-baryonic matter and modified laws of gravity. The hypothesis of existence of cold, weakly interacting nonbaryonic particle [6, 7] is subject to the correctness of general relativity (GR). However, GR is well-established experimentally within the weak conditions of solar system [8] and predictions of existence of black holes and gravitational waves [9] have also been confirmed. So far, it cannot be tested beyond the gravitational field strength larger or smaller than scales within solar system and has remained incompatible with quantum theory. Therefore, validation of GR can be contested in conditions on largest scales or on smallest scales and because $95 \%$ of the universe consists of unknown matter or energy initially unaccounted for in the theory. MOdified Newtonian Dynamics (MOND) 10 12 is presented as an alternative and proposes to replace 'dark matter' by explaining Mass Discrepancy Acceleration Relation (MDAR) [13, 14]. Recently, Super-fluid dark matter (SFDM) model was presented 2, 3, that describes a scalar particle that condenses into a superfluid. However, these models have limitations on largest scales.

According to GR, spacetime is featureless and has no intrinsic properties. It can curve and bend like a differentiable manifold or surface and is a dynamical stage on which physical phenomenon takes place. The theory has provided incredible development in our understanding of space and time where both are assumed to be dimensions with no physical rooting but this has served the theory well. Yet, there remains an enigma around understanding time and emergence of arrow of time [15]. Results in general relativity are limited to geometry of spacetime and its effects on matter. Consequently, any physical phenomenon has to be captured in terms of metric of spacetime. This is simple and elegant but limiting as well.

The mystery of the accelerated expansion [16, 17] of universe is mathematically resolved by non-zero cosmological constant but what is sourcing it and why it has unusually small value are still open questions. Negative mass spanning the interstellar space is discussed [18] as a source of 'dark energy' accelerating the universe and alternatively, SFDM models unify dark energy and dark matter. However, both models are found to have limitations. Other models propose dark matter-dark energy interactions [19, 20] as possible source.

\section{CALIBRATING EINSTEIN'S FIELD EQUATIONS}

Einstein introduced cosmological constant $(\Lambda)$ term to his field equations to formulate for a static universe. Eventually, it was dropped when universe was found to be expanding however, it was once again introduced later to compensate for accelerating universe. Thus historically observations have calibrated EFEs.

Once again, there are unexplained observations that hint at existence of new type of matter if EFEs are correct in currently accepted form. However, the model presented in the working paper establishes gravity as flow and distribution of energy and GR's spacetime as a mathematical construct that is equivalent to this new defini- 
tion of gravity in weak conditions. Thus, EFEs need to be calibrated for the additional effects predicted by the proposed model of Kaal structure.

There are a few new conditions that are introduced by the Kaal structure model:

1. Source mass binds energy density.

2. On largest scales, continuous minimization of local energy density causes repulsion.

3. Matter and energy are separate entities whose interplay gives rise to physical phenomenon

Therefore, any mathematical description of flow and distribution of Kaal energy must step from being binding on large scales to being repulsive in vast, empty space on largest scales. It has to be a continuous, smooth deformation but for simplicity it can be said there must be two sets of EFEs to completely describe the Kaal structure and thus the large scale effects.

Further, the accelerated expansion is currently described by introduction of cosmological constant term $(\Lambda)$ in EFEs. Following the proposed model of Kaal structure, continuous minimization of local energy density causes accelerated expansion however the acceleration must be monotonically decreasing. Therefore, any term attempting to explain repulsive effects in Kaal theory cannot be a constant and must be dependent on time and space coordinates i.e. we've

$$
\text { Cosmological function: } \Lambda\left(t, x^{1}, x^{2}, x^{3}\right)
$$

To arrive at a simpler model which approximates to current formulation, we can make the following assumptions:

1. expansion is isotropic

2. at any given point in time, expansion rate is independent of spatial coordinates i.e.

$$
\begin{aligned}
\Lambda\left(t, x^{1}+\delta x^{1}, x^{2}+\delta x^{2}, x^{3}+\delta x^{3}\right) & =\Lambda\left(t, x^{1}, x^{2}, x^{3}\right) \\
\Lambda(t=T) & =\text { constant } \\
\Lambda(t=T+\delta T) & <\Lambda(t=T)
\end{aligned}
$$

Thus, we can approximate $\Lambda$ to be a function of time only.

Also, because matter and energy are separate entities; at any given point in space either matter or energy exists. This implies the right hand side of EFEs i.e. source of spacetime curvature in GR, the energy-momentum tensor must correspond to only KED and its flow. Classically, energy is considered as a property of matter and the energy-momentum tensor in currently adopted EFEs corresponds to energy and momentum of source mass which are considered to have no presence beyond the physical boundaries of source mass i.e. in empty space. Though this is an incoherent description of energy of source mass in Kaal theory, the mathematical description differs only by the density and flow of BKE near a source mass.
However, on largest scales, space free from influence of matter, the RHS of EFEs must correspond only to local energy density which is monotonically decreasing thus causing expansion i.e. energy-momentum tensor is defined by cosmological function. But to comply with existing cosmological model that considers universe as perfect fluid, its energy-momentum tensor is retained, however the effect of minimization of local KED (repulsion/expansion) is the dominant source since BKE due to matter is ignored (this can be assumed considering dark energy constitutes $70 \%$ mass-energy of universe). Cosmological implications of the proposed Kaal structure are not explored in this paper.

Now, calibration to EFEs is introduced as

$$
R_{\mu \nu}-\frac{1}{2} R g_{\mu \nu}=k T_{\mu \nu}+s N_{\mu \nu}
$$

where $k=8 \pi G / c^{4}$ and $s$ is some constant. $N_{\mu \nu}$ is the energy-momentum tensor for (1) Bounded Kaal Energy (BKE) near a source mass (2) monotonically decreasing local energy density and its flow on largest scales causing repulsion. $N_{\mu \nu}$ is a non-zero entity, it can be attractive (inward flow of KED) or repulsive (minimization of KED) which can be mathematically captured by the sign (+/-) of $N_{\mu \nu}$ but is never zero.

$$
s N_{\mu \nu}= \begin{cases}+\left(s N_{\mu \nu}\right)_{B K E} & \text { large scale with source } \\ -\Lambda(t) g_{\mu \nu} & \text { largest scale }\end{cases}
$$

Thus the EFEs now take two forms, first to describe spacetime when mass interacts with Kaal structure by binding it

$$
R_{\mu \nu}-\frac{1}{2} R g_{\mu \nu}=k T_{\mu \nu}+\left(s N_{\mu \nu}\right)_{B K E}
$$

and, when on largest scale, length and time measurements reduce due to reduction in local energy density causing observed accelerated expansion

$$
R_{\mu \nu}-\frac{1}{2} R g_{\mu \nu}=k\left(T_{\mu \nu}-\frac{\Lambda(t)}{k} g_{\mu \nu}\right)
$$

As discussed earlier, the transformation between these two equations is continuous and smooth which is ignored for simplicity here. This second EFE is similar to currently adopted form with cosmological constant [8, 21] except that $\Lambda$ term is no more a constant rather a function of time coordinate. Given the large age of universe, it may be difficult to observe the reduction in accelerated expansion of universe over a short period of time such as a few decades, thus for current practical purposes $\Lambda$ can be safely approximated to a constant.

It should be noted that neither of modified EFE above is an universal equation and has its own domain of application. 


\section{Considering spatial dependence}

If the second assumption made above is ignored, which ideally should be ignored when comparing observations from near and far universe, then we have $\Lambda\left(t, x^{1}, x^{2}, x^{3}\right)$. We continue to assume isotropy and adopting spherical coordinates we have $\Lambda(t, r)$ defining decreasing local KED causing expansion. Therefore, the second modified EFE takes the form

$$
R_{\mu \nu}-\frac{1}{2} R g_{\mu \nu}=k\left(T_{\mu \nu}-\frac{\Lambda(t, r)}{k} g_{\mu \nu}\right)
$$

This could mean the expansion rate measured using a galaxy in far universe may be lower compared to expansion rate measured using a galaxy in near universe i.e. expansion rate is dependent on spatial distance between galaxies.

\section{A. $\quad N_{\mu \nu}$ for Bounded Kaal Energy}

In the working paper 1, Einstein's Reduced Equation (ERE), $R=4 \pi G \rho_{s}$ was introduced to evaluate the variation in length scales due to presence of energy of source mass or otherwise. The metric was then used to arrive at variation in time scales. In actual conditions, $\rho_{s}>0$ at all points at all times i.e. energy density is positive definite. Therefore, vacuum condition solutions that consider $\rho_{s}=0$ is an approximation and not exact solutions. In weak conditions such as Schwarzschild conditions, the solution thus arrived at is an approximation since contribution of Bounded Kaal Energy (BKE) due to source mass is ignored i.e. $N_{\mu \nu}=0$.

Consider a spherically isotropic source mass of radius, $R$. At a radial distance, $r$, the variation in time and length scales due to variation in KED (aka curvature in GR spacetime) is a result of presence of source mass and $\operatorname{KED}(\kappa)$ bounded by region extending from $R$ to $r$. Thus, there is a recursive relation in evaluating BKE at any point outside of source mass such that

$$
\kappa)_{r+\delta r}=f\left(\rho_{s}, \int_{R}^{r} \kappa_{r} \mathrm{~d} V o l\right)
$$

Also, we defined the amount of KED as

$$
\kappa=H \omega
$$

where $\mathrm{H}$ is some constant and $\omega$ is the frequency of Kaal Ripples which was arrived at using the relation $\omega=|\vec{k}|$ (in units, $c=1$ ). The wave vector for Kaal Ripples is the outcome of solution to ERE as discussed in working paper [1].
By mass-energy 22] or mass-Kaal equivalence 1], BKE can then be considered as presence of additional matter that causes 'gravitational' effects in addition to source mass as understood presently. This was described as Kaal Fluid in the working paper, which is dragged [24] by rotation of source mass. Thus, $N_{\mu \nu}$, energymomentum tensor for any infinitesimal volume element of Kaal Fluid of BKE can be defined knowing its energy density $(\kappa)$ and flow characteristics. It is simpler to consider it as ideal fluid.

In Schwarzschild conditions, flow of KED is weak and ignored, thus

$$
N_{\mu \nu}=\left(\begin{array}{cccc}
+\kappa_{\delta V o l} & 0 & 0 & 0 \\
0 & 0 & 0 & 0 \\
0 & 0 & 0 & 0 \\
0 & 0 & 0 & 0
\end{array}\right)
$$

So in vacuum conditions, even though $T_{\mu \nu}=0$, the energy component of $N_{\mu \nu}$ is always positive definite.

\section{CONCLUSION}

The model considers energy and matter as separate physical entities wherein energy is property of the proposed probabilistic three-dimensional Kaal ('dark') structure spanning space. The structure defines local length and time scales which lead to energy density being equivalent to spacetime curvature. Thus, following the new description of gravity as a result of flow and distribution of energy, two modified Einstein field equations are presented that describe the 'gravitational' effects on large (attractive) and largest (repulsive) scales. Physically, there is continuous and smooth deformation between the Kaal structure described by the two equations however for simplicity this has been ignored. The additional 'gravitational' effects near a source mass are captured by the energy-momentum tensor for the Bounded Kaal Energy (BKE) in the first modified EFE. In the second modified EFE, repulsive 'gravity' (expansion of universe) is explained by the time-dependent cosmological function $(\Lambda(t))$ assuming isotropy and spatial independence. $\Lambda(t)$ is a monotonically decreasing quantity as universe as a system tends towards a lower energy state causing expansion and thus, the expansion rate of universe must fall with time.

\section{ACKNOWLEDGMENTS}

I like to thank Dr. Mahesh and others for sharing their illuminating comments on the preceding paper. They have greatly influenced this extension of the proposed model. 
[1] Shivam Naarayan. Rippling 3-Riemannian structure describing gravity with dark matter effects. 2021. hal03275327

[2] L. Berezhiani and J. Khoury, Theory of Dark Matter Superfluidity, Phys. Rev. D92, 103510 (2015).

[3] L. Berezhiani and J. Khoury, Dark matter superfluidity and galactic dynamics, Phys. Lett. B753, 639 (2016).

[4] A. Einstein, The Foundation of the General Theory of Relativity,. Ann. Phys. (Berl.), 354, 769 (1916).

[5] F. Zwicky, The Redshift of Extragalactic Nebulae, Helv. Phys. Acta 6, 110 (1933).

[6] L. Bergstrom, Non-Baryonic Dark Matter - Observational Evidence and Detection Methods, Rep. Prog. Phys. 63, 793 (2000).

[7] G. Bertone, D. Hooper and J. Silk, Particle Dark Matter: Evidence, Candidates and Constraints, Rep. Prog. Phys. 405, 279 (2005).

[8] J. B. Hartle, in Gravity: An Introduction to Einstein's General Relativity, (Pearson, UK, 2014)

[9] LIGO Scientific Collaboration, Observation of Gravitational Waves from a Binary Black Hole Merger, Phys. Rev. Lett. 116, 061102 (2016).

[10] J. Bekenstein and M. Milgrom, Does the missing mass problem signal the break-down of Newtonian gravity?, Astrophys. J. 286, 7 (1984).

[11] M. Milgrom, MOND theory, Can. J. Phys. 93, 107 (2015).

[12] R. H. Sanders, A historical perspective on modified Newtonian dynamics, Can. J. Phys. 93, 126 (2015).

[13] S. S. McGaugh, The Mass Discrepancy-Acceleration Relation: Disk Mass and the Dark Matter Distribution, As- trophys. J. 609, 652 (2004).

[14] R. H. Sanders, Mass discrepancies in galaxies: dark matter and alternatives, The Astron Astrophys Rev 2, 1 (1990).

[15] G Hooft, Time, the arrow of time, and Quantum Mechanics, arXiv e-prints, arXiv:1804.01383 (2018).

[16] A. G. Riess, Observational Evidence from Supernovae for an Accelerating Universe and a Cosmological Constant, Astron. J. 116, 1009 (1998).

[17] S. Perlmutter, Measurements of $\Omega$ and $\Lambda$ from 42 HighRedshift Supernovae, Astron. J. 517, 565 (1999).

[18] J. S. Farnes, A Unifying Theory of Dark Energy and Dark Matter: Negative Masses and Matter Creation within a Modified $\Lambda C D M$ Framework, A\&A 620, A92 (2018).

[19] R. Mainini and S. Bonometto, Mass functions in coupled Dark Energy models, Phys. Rev. D74, 04350492 (2006).

[20] S. Das, P.S. Corasaniti and J. Khoury, Super-acceleration as Signature of Dark Sector Interaction, Phys. Rev. D73, 083509 (2006).

[21] Jayant Vishnu Narlikar, in An Introduction to Cosmology, (Cambridge University Press, New Delhi, 2018)

[22] A. Einstein, Does the Inertia of a Body depend upon its Energy-content?, Ann. Phys. (Berl.), 323, 639 (1905).

[23] FRW metric makes assumptions which are incoherent with predictions of the proposed model.

[24] Or the flow of Kaal Fluid i.e. KED causes the mass to move/rotate. Kaal structure and matter can be said to have equal relationship. 